\title{
COMPORTAMIENTO DE ZIKA Y SUS COMPLICACIONES EN EL DEPARTAMENTO DE CÓRDOBA, 2016
}

\section{BEHAVIOUR OF ZIKA AND IT'S COMPLICATIONS IN THE DEPARTMENT OF CORDOBA, 2016}

\author{
Vanessa Cañavera $M^{1}$, Shirly Cogollo J ${ }^{1}$, Álvaro Sánchez $C^{2}$ \\ Recibido para publicación: Abril 25 de 2018 - Aprobado para publicación: Mayo 30 de 2018
}

\begin{abstract}
RESUMEN
Objetivo. Determinar el comportamiento de Zika y sus complicaciones en el departamento de Córdoba, año 2016 con el fin de recomendar acciones de vigilancia epidemiológica y control de vectores que contribuyan a mitigar el efecto del evento. Materiales y métodos. Estudio descriptivo, cualicuantitativo y transversal. Se hizo revisión de bases de datos de casos nuevos de Zika y sus complicaciones. Resultados. El comportamiento de Zika en Córdoba para el año 2016 hasta la semana epidemiológica 18, evidencia el registro de 2153 casos. Con un incremento en la semana epidemiológica 4 , en la cual se notificaron 469 casos. La población femenina fue la más afectada con 1626 casos $(75,5 \%)$, de los cuales 740 se registraron en gestantes siendo Montería el municipio líder hasta la semana epidemiológica 17. El número de complicaciones con sospecha de Zika registradas en el departamento fue de 21 , de los cuales 2 casos $(9,5 \%)$ corresponden a microcefalia. El Síndrome autoinmunitario más incidente fue el Síndrome de Guillain Barré (SGB) con 8 casos $(38,1 \%)$. Conclusiones. El comportamiento de Zika en Córdoba, demuestra la relación entre el aumento complicaciones previa infección por Zika y se sustenta con investigaciones realizadas a nivel mundial. Dando importancia a la implementación de acciones de promoción y prevención que disminuyan el riesgo de complicaciones y tasas de morbimortalidad por Zika.
\end{abstract}

Palabras clave: Zika, comportamiento, complicaciones, vigilancia, control, vectores.

\begin{abstract}
Objective. Determine the behaviour of the Zika virus and its complications in the department of Cordoba, 2016, recommending epidemiological surveillance actions and control of vectors to minimize event's effect. Materials and methods. Descriptive, quali-quantitative and cross-sectional study. A search of electronic data bases of new cases of Zika and its complications was performed. Results. By 2016 until 18 epidemiologic week, the behaviour of Zika in Cordoba was 2153 cases, which has been increasing to 469 cases in reference to 4 epidemiologic week. Female population was the most affected with 1626 cases $(75,5 \%)$, of which 740 was registered in pregnant women, being Monteria the most incident municipality until 17 epidemiologic week. The incidence of complications with suspected of have had Zika, in the department was 21 cases, of which $2(9,5 \%)$ was microcephaly. The most incident neurologic syndrome was GuillainBarré syndrome with 8 cases $(38,1 \%)$. Conclusion. The behaviour of Zika in Cordoba, shows the link between complications's increasing and previous Zika infection; it is supplemented by researches worldwide. Giving importance to the implementation of prevention and promotion actions aimed at decreasing the risk of complications, morbidity and mortality in those with Zika.
\end{abstract}

Keywords: Zika, behaviour, complications, surveillance, control, vectors.

\footnotetext{
${ }^{1}$ Enfermera, Universidad de Córdoba, Montería, Córdoba

${ }^{2}$ Enfermero M.Sc \& PhD en Salud Pública. Profesor Titular Facultad Ciencias de la Salud, Universidad de Córdoba, Montería, Colombia aasanchez@correo.unicordoba.edu.co
} 


\section{INTRODUCCIÓN}

Actualmente el mundo se ha visto afectado por diversas enfermedades transmisibles. Tal es el caso del Zika (ZIKV), definido por Serra (1), como un virus RNA (perteneciente a la familia Flaviviridae y género Flavivirus), que ingresa al cuerpo humano por la picadura del mosquito aedes aegypti. En contraste, el Instituto Nacional de Salud (INS) y el Ministerio de Salud y la Protección Social (MINSALUD) (2), definen el ZIKV como una enfermedad febril, zoonótica, emergente; cuya sintomatología es inespecífica y puede confundirse con otros síndromes febriles. El ZIKV fue aislado por primera vez en 1947 en los bosques de Zika, en Uganda y se demostró en seres vivos por estudios serológicos en 1952. Hasta el 1 de diciembre de 2015, 9 países confirmaron circulación autóctona del virus, entre ellos Brasil, Colombia y Venezuela (3).

Las complicaciones relacionadas con el ZIKV sugieren trastornos neurológicos y autoinmunitarios como el SGB la microcefalia. En The Lancet (4), se publicó el primer estudio de casos y controles, donde se investigó la relación entre el ZIKV y el SGB. En el departamento de Córdoba, los casos de ZIKV ascendieron a 458 hasta febrero del 2016, de los cuales "454 son embarazadas y 4 padecen SGB" (5). La investigación determinó el comportamiento de Zika y sus complicaciones en el departamento de Córdoba, año 2016, y se recomendaron acciones de vigilancia epidemiológica y control de vectores que pudieran contribuir a mitigar el efecto del evento.

\section{MATERIALES Y MÉTODOS}

Se realizó un estudio descriptivo, de corte transversal, con abordaje cualicuantitativo, a través de un muestreo probabilístico de una base de datos de 2153 casos de ZIKV facilitada por la Secretaría de Salud Departamental Laboratorio de Salud Pública, la cual incluía 740 casos en gestantes y 21 casos de complicaciones autoinmunitarias y neurológicas registrados en el departamento Córdoba durante las primeras 18 semanas epidemiológicas del año 2016.
Con anterioridad, el grupo investigador solicitó la información a la Secretaría de Salud Departamental - Laboratorio de Salud Pública, dejando claro los requerimientos específicos de la investigación en cuestión. Se tomaron como criterios de inclusión para el estudio a las personas que desarrollaron Zika durante las 18 primeras semanas epidemiológicas de 2016, a las mujeres gestantes que desarrollaron ZIKV durante el mismo período de tiempo y personas que desarrollaron complicaciones asociadas a la previa infección por ZIKV durante ese mismo período de tiempo.

La información se recolectó durante los primeros días del mes de junio. Al personal de la Secretaría, se le explicó la importancia de la investigación y los objetivos trazados por las investigadoras. El personal de dicha institución, sugirió prudencia con la información facilitada y se abstuvo de proporcionar datos personales de los pacientes, acordando brindar a las investigadoras información sobre variables generales como: total de casos en el departamento por municipio y semana epidemiológica, casos según rango de edad (de 0 a 4 años, de 4 a 9, de 10 a 14, de 15 a 19, de 20 a 24, de 25 a 29, de 30 a 34, de 35 a 39, de 40 a 44, de 45 a 49, de 50 a 54, de 55 a 59 y mayores de 60 años), casos según sexo, casos según área de procedencia (cabecera municipal, centro poblado y rural disperso), casos en gestantes por municipio y semana epidemiológica y casos de complicaciones autoinmunitarias y neurológicas con fuerte sospecha de ZIKV por municipio.

Además, se tuvo en cuenta los resultados de estudios recientes sobre la relación del virus y complicaciones asociadas, tal como los realizados por el Laboratorio de Flavivirus del Instituto Osvaldo Cruz (Brasil), el Instituto Evandro Chagas (Belem, Brasil) (6), la Polinesia Francesa y que fue publicado en the Lancet; y el realizado por el Instituto de Investigaciones Biológicas del Trópico de la Universidad de Córdoba (7).

Para el análisis y la discusión de resultados, fue necesario relacionar dichos datos con los avances a nivel epidemiológico por parte del 
INS, a través de los boletines epidemiológicos semanales. Esto permitió dar una mirada a la realidad del departamento de Córdoba en el territorio nacional. El procesamiento de la información se realizó a través de software tales como Excel 2010 y ArcGis y se presentó en tablas, gráficas y mapas.

Con relación a los aspectos éticos, se tuvo en cuenta la declaración de Helsinki a nivel Internacional y la Resolución 8430 de 1993 expedida por el Ministerio de Salud Colombiano. Para la investigación en el área de la salud, este estudio es considerado sin riesgo. Se tuvo aval previo del Comité de Acreditación y Currículo del Programa de Enfermería de la Universidad de Córdoba y de la Secretaría de Salud Departamental - Laboratorio de Salud Pública, se respetó los derechos de autor mediante citas bibliográficas.

\section{RESULTADOS}

De los 2153 casos de ZIKV presentados en el departamento de Córdoba hasta la semana epidemiológica 18, el mayor porcentaje $(16.3 \%)$, se encontró entre el rango de edad de los 20 a 24 años evidenciando una incidencia de 84,4 casos x 10000 habitantes; y el menor porcentaje (2.1\%) se encontró entre los 55 a 59 años con una incidencia de 23,5 casos x 10000 habitantes. De los cuales la población femenina fue la más afectada con un $75.5 \%$ y una incidencia de 18,8 casos x 10000 habitantes, frente a la población masculina con un $24.4 \%$ y una incidencia de 6,1 casos x 10000 habitantes.

En este sentido, la aparición de un mayor porcentaje de casos en dicho rango de edad y grupo poblacional se relacionó con la población total de 20 a 24 años y por sexo para el 2016. Dichos datos se sustentaron con la información suministrada por De Wulf (8); en la cual la pirámide poblacional de Colombia para 2016 mostró un 4,3\% de hombres y un $4,2 \%$ de mujeres en el rango de edad de 20 a 24 años, comparado con los rangos inmediatamente anterior (15 a 19 años) y próximo (25 a 29 años). Además se observó que la población femenina en el territorio nacional, representada en un 50,6\%, superó la población masculina, representada en un $49,4 \%$.

En el boletín epidemiológico semanal correspondiente a la semana epidemiológica 18 (9), se habló del comportamiento demográfico y social de los casos de enfermedad por ZIKV en Colombia, desde la fase epidémica (semana epidemiológica 32 de 2015) hasta la semana epidemiológica 18.

El comportamiento sociodemográfico evidenció que en la variable de grupo de edad el mayor número de casos se presentó en el rango de 25 a 29 años, lo cual no coincidió con la información suministrada por la Secretaría de Salud Departamental - Laboratorio de Salud Pública. Además, en el boletín mencionado el rango de edad subsecuente con mayor número de casos fue el de 20 a 24 años reportando 10524 $(13,16 \%)$ de casos confirmados de ZIKV en el territorio nacional, de los cuales, según la información de la fuente mencionada, 352 (16,3\%) correspondían al departamento de Córdoba.

Sumado a esto, el rango de edad con menor número de casos fue el de 55 a 59 años, reportando $3033(3,79 \%)$ casos confirmados de ZIKV en el territorio nacional de los cuales, según la información suministrada por la fuente de la investigación, 46 (2,1\%) correspondían al departamento de Córdoba. Lo anterior, dió veracidad a los resultados obtenidos por medio de esta investigación puesto que los datos referentes al menor número de casos para las edades entre 55 a 59 años no discreparon de los datos a nivel nacional.

Se evidenció además que el 66,62\% de casos confirmados y sospechosos, se registraron en el sexo femenino dejando como minoría los casos en la población masculina. Del mismo modo, RECOLZIKA (10), ratificó esta información al decir que un 67,5\% de los casos se han registrado en mujeres. Ambos autores, coincidieron con los datos suministrados por la Secretaría de Salud Departamental - Laboratorio de Salud Pública en relación con el mayor y menor número de casos de enfermedad por ZIKV según sexo en el departamento. 
Esta situación, respondió al hecho de que la población femenina es objetivo de vigilancia en Salud Pública al ser quienes se embarazan. Así, el caso de una mujer embarazada que presente síntomas es notificado de forma inmediata e individual por sus manifestaciones clínicas. Por otro lado, de los 2153 casos de ZIKV presentados en el departamento de Córdoba hasta la semana epidemiológica 18, el mayor porcentaje $(71,3 \%)$, se presentó en la cabecera municipal y el menor $(10,6 \%)$ se presentó en el centro poblado.

En consideración de las ideas anteriores, en la cabecera municipal la notificación de casos confirmados por parte de las unidades primarias generadoras de datos (UPGD) a las unidades notificadoras municipales (UNM), tuvo mayor cumplimiento que en el centro poblado o el área rural dispersa, zonas en las cuales la accesibilidad a centros de salud es reducida si se compara con la cabecera municipal. Por tanto en estas zonas aunque existieron casos que por clínica manifestaron la presencia de la enfermedad, éstos se manejaron fuera de las instituciones, por lo que se evidenció el menor número de casos notificados en los subregistros.

En el departamento de Córdoba para la semana epidemiológica 18, el 22\% de casos se presentó en la semana epidemiológica 4 con una incidencia de 2,7 casos x 10000 habitantes, y el $0,05 \%$ en la semana epidemiológica 17 , con una incidencia de 0,006 casos x 10000 habitantes. En este sentido, las condiciones climáticas de la mano con la orografía del departamento y las características del vector favorecieron no sólo la reproducción, supervivencia y permanencia del mismo, sino también la aparición del mayor número de casos en la semana 4.

Las características del fenómeno del niño para el período de tiempo comprendido entre el 24 al 30 de enero (semana epidemiológica 4), favorecieron la propagación de enfermedades infecciosas y alteraciones de factores medioambientales (11). Se debe tener en cuenta que, para dicho período de tiempo el protocolo para la vigilancia en Salud Pública relacionado con la enfermedad por ZIKV, aprobado el 29 de diciembre de 2015, contaba con 4 semanas de vigencia, y la mayoría de circulares estaban vigentes desde finales de 2015 y enero de 2016. En este sentido, el acoplamiento del país y más aún de sus departamentos a la epidemia del ZIKV precisaba la creación de estrategias para prevenir y controlar la incidencia de la enfermedad y sus complicaciones.

Hacia enero de 2016, las elevadas temperaturas, no sólo en las zonas de reproducción del mosquito sino también en zonas cálidas no necesariamente lluviosas, favorecieron la propagación de la enfermedad más allá de zonas puramente tropicales o incluso más secas. Al describir la orografía de Córdoba, la gobernación del departamento (12) sustentó que éste, en el punto más elevado de la serranía de Abibe (Alto de Carrizal), se encuentra a 2200msnm. Asimismo, otras elevaciones importantes como el Alto de Carepa y el Alto de Quimarí, no superan los 2200msn. Dando relevancia a lo establecido por el INS y el MINSALUD (13) en su protocolo de vigilancia en Salud Pública relacionado con la enfermedad por ZIKV.

En este sentido, las condiciones climáticas para la semana epidemiológica 4, sumadas a las características geográficas propias del departamento, ratifican el hecho de que para ésa semana éste se halla ubicado como el quinto a nivel nacional con más casos de ZIKV y el segundo a nivel de la región Caribe (1605 casos, de los cuales 1120 fueron confirmados por laboratorio y clínica y 485 fueron sospechosos).

En el boletín epidemiológico correspondiente a la semana epidemiológica 17 (14), el cual incluye datos desde la semana 40 de 2015 hasta el 30 de abril de 2016, se sustenta que a nivel nacional, la semana 17 fue la que tuvo menor registro de casos de ZIKV, coincidiendo con la información suministrada por la fuente de la investigación, ya que para esa semana se reportó 1 caso de ZIKV (0,05\%) en el departamento. Dicho caso se presentó en una gestante en el municipio de Montería. 
Por su parte, en mujeres gestantes, hasta la semana epidemiológica 17, se presentaron 740 casos, de los cuales 58,1\% fueron en la ciudad de Montería, con una incidencia de 18,7 casos x 10000 habitantes. Considerando además que en municipios como La Apartada y Montelíbano, donde el número de casos fueron pocos, la incidencia fue mayor frente a la de Montería (20,2 casos x 10000 habitantes y 20,1 casos por 10000 habitantes respectivamente). Por otro lado el $0,1 \%$ de casos se presentó en los municipios de Purísima, Moñitos, Tuchin, Los Córdobas y Cotorra (individualmente), haciendo énfasis en Tuchin, con una incidencia de 0,5 casos x 10000 habitantes. Sumado a esto, el 24,9\% de dichos casos se presentó en la semana epidemiológica 4 con una incidencia de 2,234 casos x 10000 habitantes; y el 0,13\% en las semanas epidemiológicas 15 y 17 con una incidencia de 0,012 casos x 10000 habitantes.

De este modo, el hecho de estar embarazada en el período de tiempo correspondiente a la semana epidemiológica 4, de la mano con cambios en los factores medioambientales a nivel nacional, influyó en que los casos aumentaran al punto de ubicar a Córdoba como el cuarto departamento con mayor incidencia de casos confirmados, como lo sustentó el INS en el boletín epidemiológico de esa semana (15). La información de la Secretaría de Salud Departamental - Laboratorio de Salud Pública mostró los casos confirmados entre las semanas epidemiológicas 1 a 17 de 2016, los cuales fueron 740. Por su parte la información publicada por el INS para esa semana, ubicó a Córdoba como el tercero a nivel nacional por presentar casos de ZIKV en gestantes con 121 casos, pero no sustentó la situación del departamento para esa semana. En este sentido, ambos datos coincidieron en que fue la semana epidemiológica 4 en la que más se presentaron casos de ZIKV aunque discreparon en los datos numéricos.

Por otro lado, de los 21 casos de complicaciones neurológicas asociadas a la presencia de la enfermedad por virus ZIKV, en el departamento de Córdoba, el mayor porcentaje, correspondiente a un $38,1 \%$, se presentó en la ciudad de Montería y se evidenció que la complicación más incidente fue el SGB, mientras que el menor porcentaje $(4,8 \%)$ se presentó en los municipios de Buenavista, Canalete, Cereté, Ciénaga de oro, Los Córdobas, Sahagún, San Antero, Tierralta y Tuchín, evidenciando que las complicaciones menos incidentes fueron: degeneración del sistema nervioso no especificada, otras encefalitis virales transmitidas por mosquitos, encefalitis viral no especificada, otras deformidades adquiridas de la cabeza, polineuropatía en enfermedades infecciosas y parasitarias en otra parte, y polineuropatía inflamatoria no especificada.

Además del SGB (8 casos), las complicaciones más incidentes en el departamento fueron polineuropatía no especificada con 5 casos y microcefalia con 2. En este sentido, para ese momento epidemiológico (18 primeras semanas epidemiológicas de 2016) el municipio de Montería tuvo mayor número de mujeres gestantes con ZIKV, esto, de la mano con los cambios en los factores medioambientales, aumentó el riesgo de presentar complicaciones asociadas a la infección por ZIKV, como la microcefalia. Por su parte, en el resto de la población infectada, el presentar la enfermedad en este período de tiempo favoreció que se dieran más casos de SGB.

El INS valida la información antes mencionada en su boletín epidemiológico de la semana 18, ya que concuerda en que la complicaciones más incidentes fueron los síndromes autoinmunitarios y neurológicos, dentro de los cuales el SGB fue el que tuvo más casos (335) en el territorio nacional. Es importante resaltar que a pesar de que la mayoría de casos de ZIKV se presentaron en la población femenina a nivel nacional y departamental, la población masculina fue la que más registró casos de SGB.

\section{DISCUSIÓN}

En el boletín epidemiológico semanal correspondiente a la semana epidemiológica $18^{10}$, se habla del comportamiento demográfico y social de los casos de enfermedad por 
ZIKV en Colombia, desde la fase epidémica (semana epidemiológica 32 de 2015) hasta la semana epidemiológica 18. El comportamiento sociodemográfico evidencia que en la variable de grupo de edad el mayor número de casos se presentó en el rango de 25 a 29 años, lo cual no coincide con la información suministrada por el laboratorio de Salud Pública de Córdoba. Además, en el boletín mencionado el rango de edad subsecuente con mayor número de casos es el de 20 a 24 años reportando 10524 (13,16\%) de casos confirmados de ZIKV en el territorio nacional, de los cuales, según la información del laboratorio mencionado, 352 (16,3\%) corresponden al departamento de Córdoba.

El rango de edad con menor número de casos fue el de 55 a 59 años, reportando 3033 (3,79\%) casos confirmados de ZIKV en el territorio nacional de los cuales, según la información suministrada por la fuente de la investigación, 46 (2,1\%) corresponden al departamento de Córdoba. Lo anterior, da veracidad a los resultados obtenidos por medio de esta investigación puesto que los datos referentes al menor número de casos para las edades entre 55 a 59 años no discrepan de los datos a nivel nacional.

El $66,62 \%$ de casos confirmados y sospechosos, se registraron en el sexo femenino dejando como minoría los casos en la población masculina. Del mismo modo, RECOLZIKA ${ }^{11}$, ratifica esta información al decir que un $67,5 \%$ de los casos se han registrado en mujeres. Ambos autores, coinciden con los datos suministrados por el laboratorio de Salud Pública de Córdoba en relación con el mayor y menor número de casos de enfermedad por ZIKV según sexo en el departamento.

Esta situación, responde al hecho de que la población femenina es objetivo de vigilancia en Salud Pública al ser quienes se embarazan. Así, el caso de una mujer embarazada que presente síntomas es notificado de forma inmediata e individual por sus manifestaciones clínicas. La mayor parte de la población se encuentra en el municipio de Montería, lo cual se relaciona con el mayor número de casos según área de procedencia evidenciados en la cabecera municipal.
El fenómeno del niño para el período de tiempo comprendido entre el 24 al 30 de enero (semana epidemiológica 4), favorecieron la propagación de enfermedades infecciosas y alteraciones de factores medioambientales Hacia enero de 2016, las elevadas temperaturas, no sólo en las zonas de reproducción del mosquito sino también en zonas cálidas no necesariamente lluviosas, favorecieron la propagación de la enfermedad más allá de zonas puramente tropicales o incluso más secas ${ }^{12}$.

El INS y el MINSALUD ${ }^{13}$, a través del protocolo de vigilancia en Salud Pública relacionado con la enfermedad por ZIKV, afirma que ésta se transmite por la picadura de hembras de mosquitos del género aedes infectadas por el ZIKV presentes en áreas urbanas y algunas áreas rurales por debajo de los 2200msnm haciendo referencia a los modos de transmisión de la infección por ZIKV.

En el contexto de las ideas anteriores, al describir la orografía de Córdoba, la gobernación del departamento14 sustenta que éste, en el punto más elevado de la serranía de Abibe (Alto de Carrizal), se encuentra a 2200msnm. Asimismo, otras elevaciones importantes como el Alto de Carepa y el Alto de Quimarí, no superan los 2200msn. En este sentido, las condiciones climáticas para la semana epidemiológica 4, sumadas a las características geográficas propias del departamento, ratifican el hecho de que para ésa semana éste se halla ubicado como el quinto a nivel nacional con más casos de ZIKV y el segundo a nivel de la región Caribe (1605 casos, de los cuales 1120 fueron confirmados por laboratorio y clínica y 485 fueron sospechosos).

Se debe tener en cuenta que el boletín semanal15, que reporta los datos de la semana epidemiológica 4 incluye los casos de ZIKV desde la semana epidemiológica 40 de 2015. Por tanto se observan discrepancias entre los valores del boletín y los valores de la información suministrada por el laboratorio de Salud Pública de Córdoba, el cual sólo tiene la información de las semanas epidemiológicas 1 a 
18 de 2016. Sin embargo, ambas informaciones coinciden en el hecho de que fue la semana epidemiológica 4 la que presentó mayor número de casos para el año en cuestión.

En el boletín epidemiológico correspondiente a la semana epidemiológica 1716, el cual incluye datos desde la semana 40 de 2015 hasta el 30 de abril de 2016, se sustenta que a nivel nacional, la semana 17 fue la que tuvo menor registro de casos de ZIKV, coincidiendo con la información suministrada por la fuente de la investigación, ya que para esa semana se reportó 1 caso de ZIKV $(0,05 \%)$ en el departamento. Dicho caso se presentó en una gestante en el municipio de Montería.

Teniendo en cuenta los resultados, el mayor número de casos nuevos se presentaron en Montería, situación que responde a los reportes realizados por las instituciones de salud y mayor divulgación de la información a través de medios masivos de comunicación. Para el caso de las mujeres, el hecho de estar embarazadas en el período de tiempo correspondiente a la semana epidemiológica 4, de la mano con cambios en los factores medioambientales a nivel nacional, influyó en que los casos aumentaran al punto de ubicar a córdoba como el cuarto departamento con mayor incidencia de casos confirmados, como lo sustenta el INS en el boletín epidemiológico de esa semana. La información del laboratorio de SP de Córdoba muestra los casos confirmados entre las semanas epidemiológicas 1 a 17 de 2016, los cuales fueron 740 .

Por su parte la información publicada por el INS para esa semana, toma datos desde la fase epidémica en 2015 hasta la semana 17 de 2016, con lo cual Córdoba se ubica como el tercero a nivel nacional por presentar casos de ZIKV en gestantes con 121 casos, pero no sustenta la situación del departamento para esa semana. En este sentido, ambos datos coinciden en que fue la semana epidemiológica 4 en la que más se presentaron casos de ZIKV aunque discrepa en los datos numéricos.
Es importante mencionar, que la población femenina al ser la que se embaraza, es la más vulnerable y tiene mayor interés en SP. Del mismo modo, se debe resaltar que las condiciones geográficas del departamento, de la mano con las condiciones ambientales para ese período de tiempo y la vulnerabilidad de la población femenina en estado de gravidez, favorecieron que para dicha semana se hayan presentado el mayor número ingresos de casos al sistema de vigilancia.

EI INS valida la información antes mencionada en su boletín epidemiológico de la semana 18, ya que a pesar de que éste incluye la información desde la semana epidemiológica 42 de 2015 hasta la 18 de 2016 concuerda en que las complicaciones más incidentes fueron los síndromes neurológicos, dentro de los cuales el SGB fue el que tuvo más casos (335) en el territorio nacional. Es importante resaltar que a pesar de que la mayoría de casos de ZIKV se presentaron en la población femenina a nivel nacional y departamental, la población masculina fue la que más registró casos de síndromes neurológicos.

En el mismo boletín, en lo referente a los casos de síndromes neurológicos con antecedente de enfermedad compatible con infección por ZIKV, por entidad territorial de residencia durante la vigilancia especial en Colombia desde la semana epidemiológica 42 de 2015 a 18 de 2016, el departamento de Córdoba registró 24 casos. Esto es relacionado con la información suministrada por el laboratorio de SP, según la cual el departamento de Córdoba, registró 21 casos de complicaciones neurológicas. Éstos, son datos similares a pesar de que la información del laboratorio sólo incluye datos de 2016 y el boletín del INS incluye datos desde la semana 42 de 2015 hasta la 18 de 2016.

Se concluye que las características sociodemográficas que favorecen la aparición de complicaciones en el departamento de Córdoba se relacionan principalmente con dos situaciones: la primera, el hecho de estar o vivir en zona endémica por debajo de los $2200 \mathrm{msnm}$ y estar embarazada (riesgo de microcefalia en 
recién nacidos), y la segunda, el hecho de estar o vivir en zona endémica por debajo de los 2200msnm y presentar la infección por ZIKV (riesgo de SGB u otros síndromes neurológicos).

\section{CONCLUSIÓN}

La población femenina al ser la más numerosa en el departamento, fue la que más reportó casos confirmados de ZIKV. Además, las gestantes por su condición especial y por ser objetivo de la vigilancia en Salud Pública, presentaron un alto índice de notificación de casos, siendo Montería el municipio líder hacia la semana epidemiológica 4.

El rango de edad que mayor número de casos presentó en el departamento fue el de 20 a 24 años por ser el grupo con mayor número de personas según la pirámide poblacional de Colombia para el año 2016.

La incidencia del ZIKV en el departamento favoreció la propagación de complicaciones, dentro de las cuales la que más presentó casos fue el SGB en el municipio de Montería.

La presencia de casos de microcefalia se relaciona con el hecho de que en el departamento la población femenina es la más numerosa y por tanto, durante el embarazo, si presenta ZIKV aumenta su susceptibilidad para desarrollar complicaciones asociadas.

Las características geográficas y factores medioambientalesparticularesdeldepartamento de Córdoba, favorecen la permanencia del vector transmisor y propagación rápida de la enfermedad. Sumado a esto, la presencia de casos como microcefalia y SGB evidencian la susceptibilidad de la población cordobesa a enfermar y desarrollar complicaciones relacionadas con la previa infección por ZIKV, como lo sustenta la información suministrada por la secretaría de salud departamental.

Las características sociodemográficas que favorecen la aparición de complicaciones se relacionan principalmente con dos situaciones: la primera, el hecho de estar o vivir en zona endémica por debajo de los 2200 msnm y estar embarazada (riesgo de microcefalia en recién nacidos), y la segunda, el hecho de estar o vivir en zona endémica por debajo de los 2200msnm y presentar la infección por ZIKV (riesgo de SGB u otros síndromes neurológicos).

\section{CONFLICTO DE INTERÉS}

Los autores declaran no tener conflicto de interés.

\section{REFERENCIAS}

1. Serra, M Á. Fiebre por virus Zika: una alerta necesaria. En: REVISTA HABANERA DE CIENCIAS MÉDICAS. vol. 15, no. 1. [Disponibleen internet] http://scielo.sld.cu/ scielo.php?script=sci_arttext\&pid=S1729519X2016000100001 [Citado el 30 de abril de 2016].

2. INSTITUTO NACIONAL DE SALUD; MINISTERIO DE SALUD Y DE LA PROTECCIÓN SOCIAL. Protocolo de vigilancia en salud pública enfermedad por virus Zika. Vol. 1. 2016-01-21. PRO-R02.056 [Disponible en internet] http://www.ins.gov.co/lineas-de-accion/ Subdireccion-Vigilancia/sivigila/ Protocolos\%20SIVIGILA/PRO\%20Zika. pdf [Citado el 30 de abril de 2016].

3. Serra, M Á. Fiebre por virus Zika: una alerta necesaria. En: REVISTA HABANERA DE CIENCIAS MÉDICAS. vol. 15, no. 1. [Disponible en internet] http://scielo.sld.cu/ scielo.php?script=sci_arttext\&pid=S1729519X2016000100001 [Citado el 30 de abril de 2016].

4. Cao-lormeau, Van-Mai, Blake, Alexandre; Mons, Sandrine; et al. Guillain-barré syndrome outbreak associated with zika virus infection in french Polynesia: a case-control study. The Lancet 2016; Publicado en línea 29 de feb. http://www.thelancet.com/journals/ lancet/article/PIIS0140-6736(16)00562-6/ abstract[Citado el 30 de abril de 2016]. 
5. El Meridiano. Cuatro casos de Guillain Barré confirmados en Córdoba. 22 de febrero de 2016. EM. 2016. [Disponible en internet] http://elmeridiano.co/4-casos-de-guillain-barre-confirmados-en-cordoba/32297 [Citado el 30 de abril de 2016].

6. ORGANIZACIÓN MUNDIAL DE LA SALUD, ORGANIZACIÓN PANAMERICANA DE LA SALUD. Alerta epidemiológica, Síndrome neurológico, anomalías congénitas e infección por virus Zika. Implicaciones para la salud de las Américas 1 de diciembre de 2015. P. 1 - 3. [Disponible en internet] http://www.paho.org/hq/index. php?option $=$ com_docman \&task $=$ doc view\&Itemid=270\&gid=32404\&lang [Citado el 1 de mayo de 2016].

7. UNIVERSIDAD DE CÓRDOBA. Instituto de Investigaciones Biológicas del Trópico, realiza estudio del Guillain Barré. [Disponible en internet] http:// www.unicordoba.edu.co/index.php/ prensa/boletines/1226-instituto-deinvestigaciones-biologicas- del-tropicoiibt-realiza-estudio-del-guillain-barre [Citado el 12 de abril de 16].

8. De Wulf, M. United Nations, Department of Economic and Social Affairs, Population Division. World Population Prospects: The 2015 revision. [Disponible en internet] https://populationpyramid. net/es/colombia/2016/ [Citado el 25 de septiembre de 2016].

9. INSTITUTO NACIONAL DE SALUD. Boletín epidemiológico semanal: semana epidemiológica número 18 de 2016 (01 may, al 07 may). P. 92. [Disponible en internet] http://www.ins.gov.co/boletin-epidemiologico/Boletn\%20Epidemiolgico/ 2016\%20 Bolet $\%$ C3\%ADn\%20epidemiol\%C3\%B3gico\%20semana\%2018.pdf [Citado el 25 de septiembre de 2016].
10. (RECOLZIKA). Acta Médica Peruana. 2016; 33 (1):79-81. [Disponible en internet] http://www.scielo.org.pe/ scielo. php?pid=S172859172016000100013 \&script=sci_arttext [Citado el 28 de septiembre de 2016].

11. Picazo M. ¿Favorece el niño la propagación del Zika?. El tiempo del mundo. 30 de enero de 2016 [Disponible en internet] http://picazo.eltiempo.es/2016/01 /30/ favorece-el-nino-la-propagacion-del-zika/ [Citado el 28 de septiembre de 2016].

12. Gobernación de Córdoba. Geografía de Córdoba. [Disponible en internet] http:// www.cordoba.gov.co/cordoba/geografia. html [Citado el 28 de septiembre de 2016].

13. INSTITUTO NACIONAL DE SALUD; MINISTERIO DE SALUD Y DE LA PROTECCIÓN SOCIAL. Protocolo de vigilancia en salud pública enfermedad por virus Zika. Vol. 1. 2016-01-21. PRO-R02.056 [Disponible en internet] http://www.ins.gov.co/lineas-de-accion/ Subdireccion-Vigilancia/sivigila/ Protocolos\% 20SIVIGILA/PRO\%20Zika. pdf [Citado el 30 de abril de 2016].

14. INSTITUTO NACIONAL DE SALUD. Boletín epidemiológico semanal: semana epidemiológica número 17 de 2016 (24 abr. Al 30 abr). P. 90. [Disponible en internet] http://www.ins.gov.co/boletin-epidemiologico/Boletn\%20Epidemiolgico/ 2016\%20 Bolet $\%$ C3\%ADn\%20epidemiol\%C3\%B3 gico\%20semana\%2017.pdf [Citado el 28 de septiembre de 2016].

15. INSTITUTO NACIONAL DE SALUD. Boletín epidemiológico semanal: semana epidemiológica número 4 de 2016 (24 ene, al 30 ene). P., 45 [Disponible en internet] http://www.ins.gov.co/ boletin-epidemiologico/Boletn $\% 20$ Epidemiolgico $\quad / 2016 \% 20 B o l e t i n \% 20$ epidemiologico\%20semana\%204.pdf [Citado el 28 de septiembre de 2016]. 